\title{
Some approximation results on
} Bernstein-Schurer operators defined by $(p, q)$-integers

\author{
Mohammad Mursaleen ${ }^{1,2^{*}}$, Md Nasiruzzaman ${ }^{1}$ and Ashirbayev Nurgali ${ }^{3}$
}

\author{
"Correspondence: \\ mursaleenm@gmail.com \\ 1 Department of Mathematics, \\ Aligarh Muslim University, Aligarh, \\ 202002, India \\ ${ }^{2}$ Operator Theory and Applications \\ Research Group, Department of \\ Mathematics, Faculty of Science, \\ King Abdulaziz University, P.O. Box \\ 80203, Jeddah, 21589, Saudi Arabia \\ Full list of author information is \\ available at the end of the article
}

\begin{abstract}
Recently, Mursaleen et al. (On ( $p, q)$-analogue of Bernstein operators, arXiv:1503.07404) introduced and studied the $(p, q)$-analog of Bernstein operators by using the idea of $(p, q)$-integers. In this paper, we generalize the $q$-Bernstein-Schurer operators using $(p, q)$-integers and obtain a Korovkin type approximation theorem. Furthermore, we obtain the convergence of the operators by using the modulus of continuity and prove some direct theorems.
\end{abstract}

MSC: $41 \mathrm{~A} 10 ; 41 \mathrm{~A} 25 ; 41 \mathrm{~A} 36$

Keywords: $q$-integers; $(p, q)$-integers; Bernstein operator; $(p, q)$-Bernstein operator; $q$-Bernstein-Schurer operator; $(p, q)$-Bernstein-Schurer operator; modulus of continuity

\section{Introduction and preliminaries}

In 1912, Bernstein [1] introduced the following sequence of operators $B_{n}: C[0,1] \rightarrow C[0,1]$ defined for any $n \in \mathbb{N}$ and $f \in C[0,1]$ :

$$
B_{n}(f ; x)=\sum_{k=0}^{n}\left(\begin{array}{l}
n \\
k
\end{array}\right) x^{k}(1-x)^{n-k} f\left(\frac{k}{n}\right), \quad x \in[0,1] .
$$

By applying the idea of $q$-integers, the $q$-Bernstein operators were introduced by Lupaş [2] and later by Philip [3]. Since then, many authors introduced $q$-generalization of various operators and investigated several approximation properties. For instance, the $q$-analog of Stancu-Beta operators in [4] and [5]; the $q$-analog of Bernstein-Kantorovich operators in [6]; the $q$-Baskakov-Kantorovich operators in [7]; the $q$-Szász-Mirakjan operators in [8]; the $q$-Bleimann-Butzer-Hahn operators in [9] and in [10]; the $q$-analog of Baskakov and Baskakov-Kantorovich operators in [11]; the $q$-analog of Szász-Kantorovich operators in [12]; and the $q$-analog of generalized Bernstein-Schurer operators in [13]. Besides this, we also refer to some recent related work on this topic: e.g. [14] and [15].

First we give here some notations on the $(p, q)$-calculus.

The $(p, q)$-integer was introduced in order to generalize or unify several forms of $q$ oscillator algebras well known in the earlier physics literature related to the representation

(c) 2015 Mursaleen et al. This article is distributed under the terms of the Creative Commons Attribution 4.0 International License (http://creativecommons.org/licenses/by/4.0/), which permits unrestricted use, distribution, and reproduction in any medium, provided you give appropriate credit to the original author(s) and the source, provide a link to the Creative Commons license, and indicate if changes were made. 
theory of single parameter quantum algebras $[16]$. The $(p, q)$-integer $[n]_{p, q}$ is defined by

$$
[n]_{p, q}=\frac{p^{n}-q^{n}}{p-q}, \quad n=0,1,2, \ldots, 0<q<p \leq 1
$$

The $(p, q)$-binomial expansion is

$$
(x+y)_{p, q}^{n}:=(x+y)(p x+q y)\left(p^{2} x+q^{2} y\right) \cdots\left(p^{n-1} x+q^{n-1} y\right)
$$

and the $(p, q)$-binomial coefficients are defined by

$$
\left[\begin{array}{l}
n \\
k
\end{array}\right]_{p, q}:=\frac{[n]_{p, q} !}{[k]_{p, q} ![n-k]_{p, q} !}
$$

Details on the $(p, q)$-calculus can be found in [17]. For $p=1$, all the notions of the $(p, q)$ calculus are reduced to the $q$-calculus [18].

In 1962, Schurer [19] introduced and studied the operators $S_{m, \ell}: C[0, \ell+1] \rightarrow C[0,1]$ defined by

$$
S_{m, \ell}(f ; x)=\sum_{k=0}^{m+\ell}\left(\begin{array}{c}
m+\ell \\
k
\end{array}\right) x^{k}(1-x)^{m+\ell-k} f\left(\frac{k}{m}\right), \quad x \in[0,1],
$$

for any $m \in \mathbb{N}$ and fixed $\ell \in \mathbb{N}$.

The $q$-analog of the Bernstein-Schurer operators is defined as follows ( $c f .[20])$ :

$$
\tilde{B}_{m, \ell}(f ; q ; x)=\sum_{k=0}^{m+\ell}\left[\begin{array}{c}
m+\ell \\
k
\end{array}\right]_{q} x^{k} \prod_{s=0}^{m+\ell-k-1}\left(1-q^{s} x\right) f\left(\frac{[k]_{q}}{[m]_{q}}\right), \quad x \in[0,1],
$$

for any $m \in \mathbb{N}, f \in C[0, \ell+1]$, and fixed $\ell$.

Recently, Mursaleen et al. [21] applied $(p, q)$-calculus in approximation theory and introduced first $(p, q)$-analog of Bernstein operators. They have also introduced and studied the approximation properties of the $(p, q)$-analog of the Bernstein-Stancu operators in [22].

In this paper, we introduce the $(p, q)$-analog of these operators. We investigate some approximation properties of these operators and obtain the rate of convergence by using the modulus of continuity. We also establish some direct theorems.

\section{Construction of $(p, q)$-Bernstein-Schurer operators}

Let $0<q<p \leq 1$. We construct the class of generalized $(p, q)$-Bernstein-Schurer operators as follows:

$$
B_{m, \ell}^{p, q}(f ; x)=\sum_{k=0}^{m+\ell}\left[\begin{array}{c}
m+\ell \\
k
\end{array}\right]_{p, q} x^{k} \prod_{s=0}^{m+\ell-k-1}\left(p^{s}-q^{s} x\right) f\left(\frac{[k]_{p, q}}{[m]_{p, q}}\right), \quad x \in[0,1],
$$

for any $m \in \mathbb{N}, f \in C[0, \ell+1]$, and fixed $\ell$. Clearly, the operators defined by (2.1) are linear and positive. If we put $p=1$ in (2.1), then the $(p, q)$-Schurer operators are reduced to the $q$-Bernstein-Schurer operators. 
Lemma 2.1 For $B_{m, \ell}^{p, q}(\cdot ; \cdot)$ given by (2.1), we have the following identities:

(i) $B_{m, \ell}^{p, q}\left(e_{0} ; x\right)=1$;

(ii) $B_{m, \ell}^{p, q}\left(e_{1} ; x\right)=\frac{[m+\ell]_{p, q}}{[m] p, q}$;

(iii) $B_{m, \ell}^{p, q}\left(e_{2} ; x\right)=\frac{[m+\ell]_{p, q}(p x+(1-x))_{p, q}^{m+\ell-1}}{[m]_{p, q}^{2}} x+\frac{[m+\ell]_{p, q}[m+\ell-1]_{p, q}}{[m]_{p, q}^{2}} q x^{2}$;

(iv) $B_{m, \ell}^{p, q}\left(e_{3} ; x\right)=\frac{[m+\ell]_{p, q}}{[m]_{p, q}^{3}}\left(p^{2} x+(1-x)\right)_{p, q}^{m+\ell-1} x+q(q+2 p) \frac{[m+\ell]_{p, q}[m+\ell-1]_{p, q}}{[m]_{p, q}^{3}}(p x+$

$$
(1-x))_{p, q}^{m+\ell-2} x^{2}+\frac{[m+\ell]_{p, q}[m+\ell-1]_{p, q}[m+\ell-2]_{p, q}}{[m]_{p, q}^{3}} q^{3} x^{3}
$$

where $e_{j}(t)=t^{j}, j=0,1,2, \ldots$.

\section{Proof}

(i) For $0<q<p \leq 1$ we use the well-known identity from [21]

$$
\sum_{k=0}^{n}\left[\begin{array}{l}
n \\
k
\end{array}\right]_{p, q} x^{k} \prod_{s=0}^{n-k-1}\left(p^{s}-q^{s} x\right)=1, \quad x \in[0,1] .
$$

Suppose we choose $n=m+\ell$.

Since

$$
(1-x)_{p, q}^{m+\ell-k}=\prod_{s=0}^{m+\ell-k-1}\left(p^{s}-q^{s} x\right)
$$

we get

$$
\sum_{k=0}^{m+\ell}\left[\begin{array}{c}
m+\ell \\
k
\end{array}\right]_{p, q} x^{k} \prod_{s=0}^{m+\ell-k-1}\left(p^{s}-q^{s} x\right)=1 .
$$

Consequently, $B_{m, \ell}^{p, q}\left(e_{0} ; x\right)=1$.

(ii) Clearly we have

$$
\begin{aligned}
B_{m, \ell}^{p, q}\left(e_{1} ; x\right) & =\sum_{k=1}^{m+\ell}\left[\begin{array}{c}
m+\ell \\
k
\end{array}\right]_{p, q} x^{k} \prod_{s=0}^{m+\ell-k-1}\left(p^{s}-q^{s} x\right) \frac{[k]_{p, q}}{[m]_{p, q}} \\
& \left.=x \sum_{k=0}^{m+\ell-1}\left[\begin{array}{c}
m+\ell \\
k+1
\end{array}\right]_{p, q} x^{k} \prod_{s=0}^{m+\ell-k-2}\left(p^{s}-q^{s} x\right) \frac{[k+1]_{p, q}}{[m]_{p, q}} \quad \text { as } k \rightarrow k+1\right\} \\
& =x \frac{[m+\ell]_{p, q}}{[m]_{p, q}} \sum_{k=0}^{m+\ell-1}\left[\begin{array}{c}
m+\ell-1 \\
k
\end{array}\right]_{p, q} x^{k} \prod_{s=0}^{m+\ell-k-2}\left(p^{s}-q^{s} x\right) \\
& =x \frac{[m+\ell]_{p, q}}{[m]_{p, q}} .
\end{aligned}
$$

(iii)

$$
\begin{aligned}
B_{m, \ell}^{p, q}\left(e_{2} ; x\right) & =\sum_{k=1}^{m+\ell}\left[\begin{array}{c}
m+\ell \\
k
\end{array}\right]_{p, q} x^{k} \prod_{s=0}^{m+\ell-k-1}\left(p^{s}-q^{s} x\right) \frac{[k]_{p, q}^{2}}{[m]_{p, q}^{2}} \\
& =\frac{[m+\ell]_{p, q}}{[m]_{p, q}^{2}} \sum_{k=1}^{m+\ell}\left[\begin{array}{c}
m+\ell-1 \\
k-1
\end{array}\right]_{p, q}
\end{aligned}
$$




$$
\begin{aligned}
& \times x^{k} \prod_{s=0}^{m+\ell-k-1}\left(p^{s}-q^{s} x\right)[k]_{p, q} \quad\left\{\text { using }[k]_{p, q}^{2}=[k]_{p, q} \cdot[k]_{p, q}\right\} \\
= & \frac{[m+\ell]_{p, q}}{[m]_{p, q}^{2}} \sum_{k=1}^{m+\ell}\left[\begin{array}{c}
m+\ell-1 \\
k-1
\end{array}\right]_{p, q} \\
& \times x^{k} \prod_{s=0}^{m+\ell-k-1}\left(p^{s}-q^{s} x\right)\left(p^{k-1}+q[k-1]_{p, q}\right)
\end{aligned}
$$

by using $[k]_{p, q}=p^{k-1}+q[k-1]_{p, q}$.

Therefore we have

$$
\begin{aligned}
& B_{m, \ell}^{p, q}\left(e_{2} ; x\right)=\frac{[m+\ell]_{p, q}}{[m]_{p, q}^{2}} \sum_{k=1}^{m+\ell}\left[\begin{array}{c}
m+\ell-1 \\
k-1
\end{array}\right]_{p, q} x^{k} p^{k-1} \prod_{s=0}^{m+\ell-k-1}\left(p^{s}-q^{s} x\right) \\
& +\frac{[m+\ell]_{p, q}}{[m]_{p, q}^{2}} \sum_{k=1}^{m+\ell}\left[\begin{array}{c}
m+\ell-1 \\
k-1
\end{array}\right]_{p, q} x^{k} q[k-1]_{p, q} \prod_{s=0}^{m+\ell-k-1}\left(p^{s}-q^{s} x\right) \\
& =x \frac{[m+\ell]_{p, q}}{[m]_{p, q}^{2}} \sum_{k=0}^{m+\ell-1}\left[\begin{array}{c}
m+\ell-1 \\
k
\end{array}\right]_{p, q} x^{k} p^{k} \prod_{s=0}^{m+\ell-k-2}\left(p^{s}-q^{s} x\right) \\
& +q \frac{[m+\ell]_{p, q}}{[m]_{p, q}^{2}} \sum_{k=2}^{m+\ell}\left[\begin{array}{c}
m+\ell-1 \\
k-1
\end{array}\right]_{p, q} x^{k}[k-1]_{p, q} \prod_{s=0}^{m+\ell-k-1}\left(p^{s}-q^{s} x\right) \\
& =x \frac{[m+\ell]_{p, q}}{[m]_{p, q}^{2}} \sum_{k=0}^{m+\ell-1}\left[\begin{array}{c}
m+\ell-1 \\
k
\end{array}\right]_{p, q}(p x)^{k}(1-x)_{p, q}^{m+\ell-k-1} \\
& +q \frac{[m+\ell]_{p, q}[m+\ell-1]_{p, q}}{[m]_{p, q}^{2}} \sum_{k=2}^{m+\ell}\left[\begin{array}{c}
m+\ell-2 \\
k-2
\end{array}\right]_{p, q} x^{k} \prod_{s=0}^{m+\ell-k-1}\left(p^{s}-q^{s} x\right) \\
& =\frac{[m+\ell]_{p, q}(p x+(1-x))_{p, q}^{m+\ell-1}}{[m]_{p, q}^{2}} x \\
& +x^{2} q \frac{[m+\ell]_{p, q}[m+\ell-1]_{p, q}}{[m]_{p, q}^{2}} \sum_{k=0}^{m+\ell-2}\left[\begin{array}{c}
m+\ell-2 \\
k-2
\end{array}\right]_{p, q} \\
& \times x^{k} \prod_{s=0}^{m+\ell-k-3}\left(p^{s}-q^{s} x\right)
\end{aligned}
$$

Hence the desired result is proved.

(iv)

$$
\begin{aligned}
B_{m, \ell}^{p, q}\left(e_{3} ; x\right) & =\sum_{k=1}^{m+\ell}\left[\begin{array}{c}
m+\ell \\
k
\end{array}\right]_{p, q} x^{k} \prod_{s=0}^{m+\ell-k-1}\left(p^{s}-q^{s} x\right) \frac{[k]_{p, q}^{3}}{[m]_{p, q}^{3}} \\
& =\frac{[m+\ell]_{p, q}}{[m]_{p, q}^{3}} \sum_{k=1}^{m+\ell}\left[\begin{array}{c}
m+\ell-1]^{3} x^{k} \prod_{s=0}^{m+\ell-k-1}\left(p^{s}-q^{s} x\right)[k]_{p, q}^{2} \\
k-1
\end{array}\right]_{p, q} \\
& =\frac{[m+\ell]_{p, q}}{[m]_{p, q}^{3}} \sum_{k=1}^{m+\ell}\left[\begin{array}{c}
m+\ell-1 \\
k-1
\end{array}\right]_{p, q} x^{k} p^{2(k-1)} \prod_{s=0}^{m+\ell-k-1}\left(p^{s}-q^{s} x\right)
\end{aligned}
$$




$$
\begin{aligned}
& +\frac{[m+\ell]_{p, q}}{[m]_{p, q}^{3}} \sum_{k=1}^{m+\ell}\left[\begin{array}{c}
m+\ell-1 \\
k-1
\end{array}\right]_{p, q} x^{k} q^{2}[k-1]_{p, q}^{2} \prod_{s=0}^{m+\ell-k-1}\left(p^{s}-q^{s} x\right) \\
& +2 q \frac{[m+\ell]_{p, q}}{[m]_{p, q}^{3}} \sum_{k=1}^{m+\ell}\left[\begin{array}{c}
m+\ell-1 \\
k-1
\end{array}\right]_{p, q} x^{k} p^{k-1}[k-1]_{p, q} \prod_{s=0}^{m+\ell-k-1}\left(p^{s}-q^{s} x\right) .
\end{aligned}
$$

A small calculation shows that

$$
\begin{aligned}
& \frac{[m+\ell]_{p, q}}{[m]_{p, q}^{3}} \sum_{k=1}^{m+\ell}\left[\begin{array}{c}
m+\ell-1 \\
k-1
\end{array}\right]_{p, q} x^{k} p^{2(k-1)} \prod_{s=0}^{m+\ell-k-1}\left(p^{s}-q^{s} x\right) \\
& =x \frac{[m+\ell]_{p, q}}{[m]_{p, q}^{3}}\left(p^{2} x+(1-x)\right)_{p, q}^{m+\ell-1}, \\
& \frac{[m+\ell]_{p, q}}{[m]_{p, q}^{3}} \sum_{k=1}^{m+\ell}\left[\begin{array}{c}
m+\ell-1 \\
k-1
\end{array}\right]_{p, q} x^{k} q^{2}[k-1]_{p, q}^{2} \prod_{s=0}^{m+\ell-k-1}\left(p^{s}-q^{s} x\right) \\
& =x^{2} q^{2} \frac{[m+\ell]_{p, q}[m+\ell-1]_{p, q}}{[m]_{p, q}^{3}}(p x+(1-x))_{p, q}^{m+\ell-2} \\
& +x^{3} q^{3} \frac{[m+\ell]_{p, q}[m+\ell-1]_{p, q}[m+\ell-2]_{p, q}}{[m]_{p, q}^{3}} .
\end{aligned}
$$

Also

$$
\begin{gathered}
2 q \frac{[m+\ell]_{p, q}}{[m]_{p, q}^{3}} \sum_{k=1}^{m+\ell}\left[\begin{array}{c}
m+\ell-1 \\
k-1
\end{array}\right]_{p, q} x^{k} p^{k-1}[k-1]_{p, q} \prod_{s=0}^{m+\ell-k-1}\left(p^{s}-q^{s} x\right) \\
=2 p q x^{2} \frac{[m+\ell]_{p, q}[m+\ell-1]_{p, q}}{[m]_{p, q}^{3}}(p x+(1-x))_{p, q}^{m+\ell-2} .
\end{gathered}
$$

This completes the proof.

Lemma 2.2 Let $B_{m, \ell}^{p, q}(\cdot ; \cdot)$ be given by (2.1). Then, for any $x \in[0,1]$ and $0<q<p \leq 1$, we have the following identities:

(i) $B_{m, \ell}^{p, q}\left(e_{1}-1 ; x\right)=\frac{[m+\ell]_{p, q}}{[m]_{p, q}} x-1$;

(ii) $B_{m, \ell}^{p, q}\left(e_{1}-x ; x\right)=\left(\frac{[m+\ell]_{p, q}}{[m]_{p, q}}-1\right) x$;

(iii)

$$
\begin{aligned}
B_{m, \ell}^{p, q}\left(\left(e_{1}-x\right)^{2} ; x\right) & \\
= & \frac{[m+\ell]_{p, q}}{[m]_{p, q}^{2}}(p x+(1-x))_{p, q}^{m+\ell-1} x \\
& +\left(\left(\frac{[m+\ell]_{p, q}}{[m]_{p, q}}-1\right)^{2}+\frac{[m+\ell]_{p, q}}{[m]_{p, q}^{2}}\left(q[m+\ell-1]_{p, q}-[m+\ell]_{p, q}\right)\right) x^{2} .
\end{aligned}
$$

\section{On the convergence of $(p, q)$-Bernstein-Schurer operators}

Let $f \in C[0, \gamma]$. The modulus of continuity of $f$, denoted by $\omega(f, \delta)$, gives the maximum oscillation of $f$ in any interval of length not exceeding $\delta>0$ and it is given by the relation

$$
\omega(f, \delta)=\sup _{|y-x| \leq \delta}|f(y)-f(x)|, \quad x, y \in[0, \gamma] .
$$


It is well known that $\lim _{\delta \rightarrow 0+} \omega(f, \delta)=0$ for $f \in C[0, \gamma]$ and for any $\delta>0$ one has

$$
|f(y)-f(x)| \leq\left(\frac{|y-x|}{\delta}+1\right) \omega(f, \delta) .
$$

For $q \in(0,1)$ and $p \in(q, 1]$ obviously we have $\lim _{m \rightarrow \infty}[m]_{p, q}=\frac{1}{p-q}$. In order to obtain the convergence results of the operator $B_{m, \ell}^{p, q}$, we take a sequence $q_{m} \in(0,1)$ and $p_{m} \in\left(q_{m}, 1\right]$ such that $\lim _{m \rightarrow \infty} p_{m}=1$ and $\lim _{m \rightarrow \infty} q_{m}=1$, so we get $\lim _{m \rightarrow \infty}[m]_{p_{m}, q_{m}}=\infty$.

Theorem 3.1 Let $p=p_{m}, q=q_{m}$ satisfying $0<q_{m}<p_{m} \leq 1$ such that $\lim _{m \rightarrow \infty} p_{m}=1$, $\lim _{m \rightarrow \infty} q_{m}=1$. Then for each $f \in C[0, \ell+1]$,

$$
\lim _{m \rightarrow \infty} B_{m, \ell}^{p_{m}, q_{m}}(f ; x)=f
$$

is uniformly on $[0,1]$.

Proof The proof is based on the well-known Korovkin theorem regarding the convergence of a sequence of linear and positive operators, so it is enough to prove the conditions

$$
B_{m, \ell}^{p_{m}, q_{m}}\left(e_{j} ; x\right)=x^{j}, \quad j=0,1,2,\{\text { as } m \rightarrow \infty\}
$$

uniformly on $[0,1]$.

Clearly we have

$$
\lim _{m \rightarrow \infty} B_{m, \ell}^{p_{m}, q_{m}}\left(e_{0} ; x\right)=1
$$

By making a simple calculation we get

$$
\lim _{m \rightarrow \infty} \frac{[m+\ell]_{p_{m}, q_{m}}}{[m]_{p_{m}, q_{m}}}=1, \quad \text { as } 0<q_{m}<p_{m} \leq 1
$$

Since $0<q_{m}<p_{m} \leq 1$, we get

$$
\lim _{m \rightarrow \infty} \frac{[m+\ell]_{p_{m}, q_{m}}}{[m]_{p_{m}, q_{m}}^{2}}=0
$$

Hence we have

$$
\begin{aligned}
& \lim _{m \rightarrow \infty} B_{m, \ell}^{p_{m}, q_{m}}\left(e_{1} ; x\right)=x, \\
& \lim _{m \rightarrow \infty} B_{m, \ell}^{p_{m}, q_{m}}\left(e_{2} ; x\right)=x^{2} .
\end{aligned}
$$

Theorem 3.2 Iff $\in C[0, \ell+1]$, then

$$
\left|B_{m, \ell}^{p, q}(f ; x)-f(x)\right| \leq 2 \omega_{f}\left(\delta_{m}\right),
$$


where

$$
\begin{aligned}
\delta_{m}= & x\left|\frac{[m+\ell]_{p, q}}{[m]_{p, q}}-1\right| \\
& +\sqrt{\frac{[m+\ell]_{p, q}}{[m]_{p, q}}} \cdot \sqrt{\frac{x^{2}\left(q[m+\ell-1]_{p, q}-[m+\ell]_{p, q}\right)+x(p x+(1-x))_{p, q}^{m+\ell-1}}{[m]_{p, q}}} .
\end{aligned}
$$

Proof

$$
\begin{aligned}
\left|B_{m, \ell}^{p, q}(f ; x)-f(x)\right| & \leq \sum_{k=0}^{m+\ell}\left[\begin{array}{c}
m+\ell \\
k
\end{array}\right]_{p, q} x^{k} \prod_{s=0}^{m+\ell-k-1}\left(p^{s}-q^{s} x\right)\left|f\left(\frac{[k]_{p, q}}{[m]_{p, q}}\right)-f(x)\right| \\
& \leq \sum_{k=0}^{m+\ell}\left[\begin{array}{c}
m+\ell \\
k
\end{array}\right]_{p, q} x^{k} \prod_{s=0}^{m+\ell-k-1}\left(p^{s}-q^{s} x\right)\left(\frac{\mid[k]_{p, q}}{[m]_{p, q}-x \mid}+1\right) \omega(f, \delta) .
\end{aligned}
$$

By using the Cauchy inequality and Lemma 2.1, we have

$$
\begin{aligned}
& \left|B_{m, \ell}^{p, q}(f ; x)-f(x)\right| \\
& \leq\left(\frac{1}{\delta}\left\{\sum_{k=0}^{m+\ell}\left[\begin{array}{c}
m+\ell \\
k
\end{array}\right]_{p, q} x^{k}\left(\frac{[k]_{p, q}}{[m]_{p, q}}-x\right) \prod_{s=0}^{2 m+\ell-k-1}\left(p^{s}-q^{s} x\right)\right\}^{\frac{1}{2}}+1\right) \omega(f, \delta) \\
& =\left\{\frac{1}{\delta}\left(B_{m, \ell}^{p, q}\left(e_{2} ; x\right)-2 x B_{m, \ell}^{p, q}\left(e_{1} ; x\right)+x^{2} B_{m, \ell}^{p, q}\left(e_{0} ; x\right)\right)^{\frac{1}{2}}+1\right\} \omega(f, \delta) \\
& =\left\{\frac { 1 } { \delta } \left(\frac{[m+\ell]_{p, q}(p x+(1-x))_{p, q}^{m+\ell-1}}{[m]_{p, q}^{2}} x\right.\right. \\
& \left.\left.+x^{2}\left(\frac{[m+\ell]_{p, q}[m+\ell-1]_{p, q}}{[m]_{p, q}^{2}} q-2 \frac{[m+\ell]_{p, q}}{[m]_{p, q}}+1\right)\right)^{\frac{1}{2}}+1\right\} \omega(f, \delta) \\
& =\left\{\frac { 1 } { \delta } \left(\left(x\left(\frac{[m+\ell]_{p, q}}{[m]_{p, q}}-1\right)\right)^{2}\right.\right. \\
& +\left(\sqrt{\frac{[m+\ell]_{p, q}}{[m]_{p, q}}}\right. \\
& \left.\left.\left.\cdot \sqrt{\frac{x^{2}\left(q[m+\ell-1]_{p, q}-[m+\ell]_{p, q}\right)+x(p x+(1-x))_{p, q}^{m+\ell-1}}{[m]_{p, q}}}\right)^{2}\right)^{\frac{1}{2}}+1\right\} \omega(f, \delta) \\
& \leq\left\{\frac { 1 } { \delta } \left(x\left|\frac{[m+\ell]_{p, q}}{[m]_{p, q}}-1\right|\right.\right. \\
& +\sqrt{\frac{[m+\ell]_{p, q}}{[m]_{p, q}}} \\
& \left.\left.\cdot \sqrt{\frac{x^{2}\left(q[m+\ell-1]_{p, q}-[m+\ell]_{p, q}\right)+x(p x+(1-x))_{p, q}^{m+\ell-1}}{[m]_{p, q}}}\right)+1\right\} \omega(f, \delta),
\end{aligned}
$$

by using $\left(a^{2}+b^{2}\right)^{\frac{1}{2}} \leq(|a|+|b|)$.

Hence we obtain the desired result by choosing $\delta=\delta_{m}$. 


\section{Direct theorems on $(p, q)$-Bernstein-Schurer operators}

The Peetre $K$-functional is defined by

$$
K_{2}(f, \delta)=\inf \left\{\left(\|f-g\|+\delta\left\|g^{\prime \prime}\right\|\right): g \in \mathcal{W}^{2}\right\}
$$

where

$$
\mathcal{W}^{2}=\left\{g \in C[0, \ell+1]: g^{\prime}, g^{\prime \prime} \in C[0, \ell+1]\right\}
$$

Then there exists a positive constant $\mathcal{C}>0$ such that $K_{2}(f, \delta) \leq \mathcal{C} \omega_{2}\left(f, \delta^{\frac{1}{2}}\right), \delta>0$, where the second order modulus of continuity is given by

$$
\omega_{2}\left(f, \delta^{\frac{1}{2}}\right)=\sup _{0<h<\delta^{\frac{1}{2}}} \sup _{x \in[0, \ell+1]}|f(x+2 h)-2 f(x+h)+f(x)| .
$$

Theorem 4.1 Let $f \in C[0, \ell+1], g^{\prime} \in C[0, \ell+1]$ and satisfying $0<q<p \leq 1$. Then for all $n \in \mathbb{N}$ there exists a constant $\mathcal{C}>0$ such that

$$
\left|B_{m, \ell}^{p, q}(f ; x)-f(x)-x g^{\prime}(x)\left(\frac{[m+\ell-1]_{p, q}}{[m]_{p, q}}-1\right)\right| \leq \mathcal{C} \omega_{2}\left(f, \delta_{m}(x)\right),
$$

where

$$
\begin{aligned}
\delta_{m}^{2}(x)= & \frac{[m+\ell-1]_{p, q}}{[m]_{p, q}^{2}}(p x+(1-x))_{p, q}^{m+\ell-1} x \\
& +\left(\left(\frac{[m+\ell]_{p, q}}{[m]_{p, q}}-1\right)^{2}+\frac{[m+\ell]_{p, q}}{[m]_{p, q}^{2}}\left(q[m+\ell-1]_{p, q}-[m+\ell]_{p, q}\right)\right) x^{2} .
\end{aligned}
$$

Proof Let $g \in \mathcal{W}^{2}$. Then from the Taylor expansion, we get

$$
g(t)=g(x)+g^{\prime}(x)(t-x)+\int_{x}^{t}(t-u) g^{\prime \prime}(u) \mathrm{d} u, \quad t \in[0, \mathcal{A}], \mathcal{A}>0 .
$$

Now by Lemma 2.1, we have

$$
\begin{aligned}
B_{m, \ell}^{p, q}(g ; x)=g(x)+x g^{\prime}(x)\left(\frac{[m+\ell]_{p, q}}{[m]_{p, q}}-1\right)+ & B_{m, \ell}^{p, q}\left(\int_{x}^{t}\left(e_{1}-u\right) g^{\prime \prime}(u) \mathrm{d} u ; p, q ; x\right), \\
\left|B_{m, \ell}^{p, q}(g ; x)-g(x)-x g^{\prime}(x)\left(\frac{[m+\ell]_{p, q}}{[m]_{p, q}}-1\right)\right| & \leq B_{m, \ell}^{p, q}\left(\left|\int_{x}^{t}\right|\left(e_{1}-u\right)|| g^{\prime \prime}(u)|\mathrm{d} u ; p, q ; x|\right) \\
& \leq B_{m, \ell}^{p, q}\left(\left(e_{1}-x\right)^{2} ; p, q ; x\right)\left\|g^{\prime \prime}\right\| .
\end{aligned}
$$

Hence we get

$$
\begin{gathered}
\left|B_{m, \ell}^{p, q}(g ; x)-g(x)-x g^{\prime}(x)\left(\frac{[m+\ell]_{p, q}}{[m]_{p, q}}-1\right)\right| \\
\leq\left\|g^{\prime \prime}\right\|\left(\frac{[m+\ell]_{p, q}}{[m]_{p, q}^{2}}(p x+(1-x))_{p, q}^{m+\ell-1} x\right.
\end{gathered}
$$




$$
\left.+\left(\left(\frac{[m+\ell]_{p, q}}{[m]_{p, q}}-1\right)^{2}+\frac{[m+\ell]_{p, q}}{[m]_{p, q}^{2}}\left(q[m+\ell-1]_{p, q}-[m+\ell]_{p, q}\right)\right) x^{2}\right) .
$$

On the other hand we have

$$
\begin{aligned}
& \left|B_{m, \ell}^{p, q}(f ; x)-f(x)-x g^{\prime}(x)\left(\frac{[m+\ell-1]_{p, q}}{[m]_{p, q}}-1\right)\right| \\
& \leq\left|B_{m, \ell}^{p, q}((f-g) ; x)-(f-g)(x)\right| \\
& \quad+\left|B_{m, \ell}^{p, q}(g ; x)-g(x)-x g^{\prime}(x)\left(\frac{[m+\ell-1]_{p, q}}{[m]_{p, q}}-1\right)\right| .
\end{aligned}
$$

Since

$$
\left|B_{m, \ell}^{p, q}(f ; x)\right| \leq\|f\|
$$

we have

$$
\begin{aligned}
\left|B_{m, \ell}^{p, q}(f ; x)-f(x)-x g^{\prime}(x)\left(\frac{[m+\ell-1]_{p, q}}{[m]_{p, q}}-1\right)\right| \\
\leq\|f-g\| \\
\quad+\left\|g^{\prime \prime}\right\|\left(\frac{[m+\ell]_{p, q}}{[m]_{p, q}^{2}}(p x+(1-x))_{p, q}^{m+\ell-1} x\right. \\
\left.\quad+\left(\left(\frac{[m+\ell]_{p, q}}{[m]_{p, q}}-1\right)^{2}+\frac{[m+\ell]_{p, q}}{[m]_{p, q}^{2}}\left(q[m+\ell-1]_{p, q}-[m+\ell]_{p, q}\right)\right) x^{2}\right) .
\end{aligned}
$$

Now taking the infimum on the right hand side over all $g \in \mathcal{W}^{2}$, we get

$$
\left|B_{m, \ell}^{p, q}(f ; x)-f(x)-x g^{\prime}(x)\left(\frac{[m+\ell-1]_{p, q}}{[m]_{p, q}}-1\right)\right| \leq \mathcal{C} K_{2}\left(f, \delta_{m}^{2}(x)\right) .
$$

In view of the property of the $K$-functional, we get

$$
\left|B_{m, \ell}^{p, q}(f ; x)-f(x)-x g^{\prime}(x)\left(\frac{[m+\ell-1]_{p, q}}{[m]_{p, q}}-1\right)\right| \leq \mathcal{C} \omega_{2}\left(f, \delta_{m}(x)\right) .
$$

This completes the proof.

Theorem 4.2 Let $f \in C[0, \ell+1]$ be such that $f^{\prime}, f^{\prime \prime} \in C[0, \ell+1]$, and the sequence $\left\{p_{m}\right\},\left\{q_{m}\right\}$ satisfying $0<q_{m}<p_{m} \leq 1$ such that $p_{m} \rightarrow 1, q_{m} \rightarrow 1$ and $p_{m}^{m} \rightarrow \alpha, q_{m}^{m} \rightarrow \beta$ as $m \rightarrow \infty$, where $0 \leq \alpha, \beta<1$. Then

$$
\lim _{m \rightarrow \infty}[m]_{p_{m}, q_{m}}\left(B_{m, \ell}^{p_{m}, q_{m}}(f ; x)-f(x)\right)=\frac{x(\lambda-\alpha x)}{2} f^{\prime \prime}(x)
$$

is uniform on $[0, \ell+1]$, where $0<\lambda \leq 1$.

Proof From the Taylor formula, we have

$$
f(t)=f(x)+f^{\prime}(x)(t-x)+\frac{1}{2} f^{\prime \prime}(x)(t-x)^{2}+r(t, x)\left(e_{1}-x\right)^{2},
$$


where $r(t, x)$ is the remainder term and $\lim _{t \rightarrow x} r(t, x)=0$, therefore we have

$$
\begin{aligned}
& {[m]_{p_{m}, q_{m}}\left(B_{m, \ell}^{p_{m}, q_{m}}(f ; x)-f(x)\right)} \\
& =[m]_{p_{m}, q_{m}}\left(f^{\prime}(x) B_{m, \ell}^{p_{m}, q_{m}}\left(\left(e_{1}-x\right) ; x\right)+\frac{f^{\prime \prime}(x)}{2} B_{m, \ell}^{p_{m}, q_{m}}\left(\left(e_{1}-x\right)^{2} ; x\right)\right. \\
& \left.\quad+B_{m, \ell}^{p_{m}, q_{m}}\left(r(t, x)(t-x)^{2} ; x\right)\right) .
\end{aligned}
$$

Now by applying the Cauchy-Schwartz inequality, we have

$$
B_{m, \ell}^{p_{m}, q_{m}}\left(r(t, x)(t-x)^{2} ; x\right) \leq \sqrt{B_{m, \ell}^{p_{m}, q_{m}}\left(r^{2}(t, x) ; x\right)} \cdot \sqrt{B_{m, \ell}^{p_{m}, q_{m}}\left((t-x)^{4} ; x\right)} .
$$

Since $r^{2}(x, x)=0$, and $r^{2}(t, x) \in C[0, \ell+1]$, from Theorem 3.1, we have

$$
B_{m, \ell}^{p_{m}, q_{m}}\left(r^{2}(t, x) ; x\right)=r^{2}(x, x)=0,
$$

which implies that

$$
\begin{aligned}
& B_{m, \ell}^{p_{m}, q_{m}}\left(r(t, x)(t-x)^{2} ; x\right)=0, \\
& \lim _{m \rightarrow \infty}[m]_{p_{m}, q_{m}}\left(B_{m, \ell}^{p_{m}, q_{m}}\left(\left(e_{1}-x\right) ; x\right)\right)=x \lim _{m \rightarrow \infty}[m]_{p_{m}, q_{m}}\left(\frac{[m+\ell]_{p_{m}, q_{m}}}{[m]_{p_{m}, q_{m}}}-1\right)=0, \\
& \lim _{m \rightarrow \infty}[m]_{p_{m}, q_{m}}\left(B_{m, \ell}^{p_{m}, q_{m}}\left(\left(e_{1}-x\right)^{2} ; x\right)\right) \\
& =x \lim _{m \rightarrow \infty}[m]_{p_{m}, q_{m}} \frac{[m+\ell]_{p_{m}, q_{m}}}{[m]_{p_{m}, q_{m}}^{2}}\left(p_{m} x+(1-x)\right)_{p_{m}, q_{m}}^{m+\ell-1} \\
& \quad+x^{2} \lim _{m \rightarrow \infty}[m]_{p_{m}, q_{m}}\left(\left(\frac{[m+\ell]_{p_{m}, q_{m}}}{[m]_{p_{m}, q_{m}}}-1\right)^{2}\right. \\
& \left.\quad+\frac{[m+\ell]_{p_{m}, q_{m}}}{[m]_{p_{m}, q_{m}}^{2}}\left(q_{m}[m+\ell-1]_{p_{m}, q_{m}}-[m+\ell]_{p_{m}, q_{m}}\right)\right) \\
& \lim _{m \rightarrow \infty}[m]_{p_{m}, q_{m}}\left(B_{m, \ell}^{p_{m}, q_{m}}\left(\left(e_{1}-x\right)^{2} ; x\right)\right)=\lambda x-\alpha x^{2}=x(\lambda-\alpha x),
\end{aligned}
$$

where $\lambda \in(0,1]$ depends on the sequence $\left\{p_{m}\right\}$.

Hence we have

$$
\lim _{m \rightarrow \infty}[m]_{p_{m}, q_{m}}\left(B_{m, \ell}^{p_{m}, q_{m}}(f ; x)-f(x)\right)=\frac{x(\lambda-\alpha x)}{2} f^{\prime \prime}(x) .
$$

This completes the proof.

Now we give the rate of convergence of the operators $B_{m, \ell}^{p, q}(f ; x)$ in terms of the elements of the usual Lipschitz class $\operatorname{Lip}_{M}(v)$.

Let $f \in C[0, m+\ell], M>0$ and $0<v \leq 1$. We recall that $f$ belongs to the class $\operatorname{Lip}_{M}(v)$ if the inequality

$$
|f(t)-f(x)| \leq M|t-x|^{\nu} \quad(t, x \in(0,1])
$$

is satisfied. 
Theorem 4.3 Let $0<q<p \leq 1$. Then for each $f \in \operatorname{Lip}_{M}(v)$ we have

$$
\left|B_{m, \ell}^{p, q}(f ; x)-f(x)\right| \leq M \delta_{m}^{\nu}(x),
$$

where

$$
\begin{aligned}
\delta_{m}^{2}(x)= & \frac{[m+\ell]_{p, q}}{[m]_{p, q}^{2}}(p x+(1-x))_{p, q}^{m+\ell-1} x \\
& +\left(\left(\frac{[m+\ell]_{p, q}}{[m]_{p, q}}-1\right)^{2}+\frac{[m+\ell]_{p, q}}{[m]_{p, q}^{2}}\left(q[m+\ell-1]_{p, q}-[m+\ell]_{p, q}\right)\right) x^{2} .
\end{aligned}
$$

Proof By the monotonicity of the operators $B_{m, \ell}^{p, q}(f ; x)$, we can write

$$
\begin{aligned}
\left|B_{m, \ell}^{p, q}(f ; x)-f(x)\right| & \leq B_{m, \ell}^{p, q}(|f(t)-f(x)| ; p, q ; x) \\
& \leq \sum_{k=0}^{m+\ell}\left[\begin{array}{c}
m+\ell \\
k
\end{array}\right]_{p, q} x^{k} \prod_{s=0}^{m+\ell-k-1}\left(p^{s}-q^{s} x\right)\left|f\left(\frac{[k]_{p, q}}{[m]_{p, q}}\right)-f(x)\right| \\
& \leq M \sum_{k=0}^{m+\ell}\left[\begin{array}{c}
m+\ell \\
k
\end{array}\right]_{p, q} x^{k} \prod_{s=0}^{m+\ell-k-1}\left(p^{s}-q^{s} x\right)\left|\frac{[k]_{p, q}}{[m]_{p, q}}-x\right|^{v} \\
& =M \sum_{k=0}^{m+\ell}\left(\mathcal{P}_{m, \ell, k}(x)\left(\frac{[k]_{p, q}}{[m]_{p, q}}-x\right)^{2}\right)^{\frac{v}{2}} \mathcal{P}_{m, \ell, k}^{\frac{2-v}{2}}(x),
\end{aligned}
$$

where $\mathcal{P}_{m, \ell, k}(x)=\left[\begin{array}{c}m+\ell \\ k\end{array}\right]_{p, q} x^{k} \prod_{s=0}^{m+\ell-k-1}\left(p^{s}-q^{s} x\right)$.

Now applying the Hölder inequality, we have

$$
\begin{aligned}
\left|B_{m, \ell}^{p, q}(f ; x)-f(x)\right| & \leq M\left(\sum_{k=0}^{m+\ell} \mathcal{P}_{m, \ell, k}(x)\left(\frac{[k]_{p, q}}{[m]_{p, q}}-x\right)^{2}\right)^{\frac{v}{2}}\left(\sum_{k=0}^{m+\ell} \mathcal{P}_{m, \ell, k}(x)\right)^{\frac{2-v}{2}} \\
& =M\left(B_{m, \ell}^{p, q}\left(\left(e_{1}-x\right)^{2} ; x\right)\right)^{\frac{v}{2}} .
\end{aligned}
$$

Choosing $\delta: \delta_{m}(x)=\sqrt{B_{m, \ell}^{p, q}\left(\left(e_{1}-x\right)^{2} ; x\right)}$, we obtain

$$
\left|B_{m, \ell}^{p, q}(f ; x)-f(x)\right| \leq M \delta_{m}^{\nu}(x) .
$$

Hence, the desired result is obtained.

\section{Conclusion}

By using the notion of $(p, q)$-integers we introduced $(p, q)$-Bernstein-Schurer operators and investigated some approximation properties of these operators. We obtained the rate of convergence by using the modulus of continuity and also established some direct theorems. These results generalize the approximation results proved for $q$-Bernstein-Schurer operators, which are directly obtained by our results for $p=1$. 
Authors' contributions

The authors contributed equally and significantly in writing this paper. All authors read and approved the final manuscript.

\section{Author details}

${ }^{1}$ Department of Mathematics, Aligarh Muslim University, Aligarh, 202002, India. ${ }^{2}$ Operator Theory and Applications Research Group, Department of Mathematics, Faculty of Science, King Abdulaziz University, P.O. Box 80203, Jeddah, 21589 Saudi Arabia. ${ }^{3}$ Science-Pedagogical Faculty, M. Auezov South Kazakhstan State University, Shymkent, 160012, Kazakhstan.

\section{Acknowledgements}

The second author (MN) acknowledges the financial support of UGC BSR Fellowship, and the third author (AN) gratefully acknowledges the financial support from M. Auezov South Kazakhstan State University, Shymkent.

\section{Received: 27 April 2015 Accepted: 23 July 2015 Published online: 13 August 2015}

\section{References}

1. Bernstein, SN: Démostration du théorèeme de Weierstrass fondée sur le calcul de probabilités. Commun. Soc. Math. Kharkow (2) 13, 1-2 (1912/1913)

2. Lupaş, A: A q-analogue of the Bernstein operator. Semin. Numer. Stat. Calc., Univ. Babeş-Bolyai 9, 85-92 (1987)

3. Philips, GM: On generalized Bernstein polynomials. In: Griffits, DF, Watson, GA (eds.) Numerical Analysis: AR Mitchell 75th Birthday Volume pp. 263-269. World Scientific, Singapore (1996)

4. Aral, A, Gupta, V: On q-analogue of Stancu-beta operators. Appl. Math. Lett. 25, 67-71 (2012)

5. Mursaleen, $M$, Khan, A: Statistical approximation properties of modified $q$-Stancu-Beta operators. Bull. Malays. Math. Soc. 36(3), 683-690 (2013)

6. Radu, C: Statistical approximation properties of Kantorovich operators based on $q$-integers. Creative Math. Inform 17(2), 75-84 (2008)

7. Gupta, V, Radu, C: Statistical approximation properties of q-Baskokov-Kantorovich operators. Cent. Eur. J. Math. 7(4), 809-818 (2009)

8. Örkcü, M, Doğru, O: Weighted statistical approximation by Kantorovich type $q$-Szász Mirakjan operators. Appl. Math Comput. 217, 7913-7919 (2011)

9. Aral, A, Doğru, O: Bleimann Butzer and Hahn operators based on q-integers. J. Inequal. Appl. 2007, 79410 (2007)

10. Ersan, S, Doğru, O: Statistical approximation properties of q-Bleimann, Butzer and Hahn operators. Math. Comput. Model. 49, 1595-1606 (2009)

11. Mahmudov, NI: Statistical approximation of Baskakov and Baskakov-Kantorovich operators based on the $q$-integers Cent. Eur. J. Math. 8(4), 816-826 (2010)

12. Mahmudov, NI, Gupta, V: On certain q-analogue of Szász Kantorovich operators. J. Appl. Math. Comput. 37, 407-419 (2011)

13. Mursaleen, $M$, Khan, A: Generalized $q$-Bernstein-Schurer operators and some approximation theorems. J. Funct. Spaces Appl. 2013, 719834 (2013). doi:10.1155/2013/719834

14. Mishra, VN, Khatri, K, Mishra, LN, Deepmala: Inverse result in simultaneous approximation by Baskakov-Durrmeyer-Stancu operators. J. Inequal. Appl. 2013, 586 (2013)

15. Mohiuddine, SA, Alotaibi, A: Korovkin second theorem via statistical summability (C, 1). J. Inequal. Appl. 2013, 149 (2013)

16. Chakrabarti, $R$, Jagannathan, $R: A(p, q)$-oscillator realization of two parameter quantum algebras. J. Phys. A, Math. Gen. 24, 711-718 (1991)

17. Sahai, V, Yadav, S: Representations of two parameter quantum algebras and p, $q$-special functions. J. Math. Anal. Appl. 335, 268-279 (2007)

18. Andrews, GE, Askey, R, Roy, R: Special Functions. Encyclopedia of Mathematics and Its Applications, vol. 71. Cambridge University Press, Cambridge (1999)

19. Schurer, F: Linear positive operators in approximation theory. Math. Inst. Techn. Univ. Delft Report (1962)

20. Muraru, CV: Note on q-Bernstein-Schurer operators. Stud. Univ. Babeş-Bolyai, Math. 56(2), 489-495 (2011)

21. Mursaleen, M, Ansari, KJ, Khan, A: On ( $p, q)$-analogue of Bernstein operators. Appl. Math. Comput. 266, 874-882 (2015). doi:10.1016/j.amc.2015.04.090

22. Mursaleen, $\mathrm{M}$, Ansari, KJ, Khan, A: Some approximation results by $(p, q)$-analogue of Bernstein-Stancu operators. Appl. Math. Comput. 264, 392-402 (2015). doi:10.1016/j.amc.2015.03.135

\section{Submit your manuscript to a SpringerOpen ${ }^{\ominus}$ journal and benefit from:}

- Convenient online submission

- Rigorous peer review

Immediate publication on acceptance

- Open access: articles freely available online

- High visibility within the field

- Retaining the copyright to your article 